\title{
Surface acoustic wave band gaps in micro-machined air/silicon phononic structures - theoretical calculation and experiment
}

\author{
Tsung-Tsong Wu* Zi-Gui Huang and Shih-Yang Liu \\ Institute of Applied Mechanics, National Taiwan University, Taipei 106, Taiwan
}

Received June 16, 2004; accepted September 9, 2004

\section{Phononic crystal / SAW (Surface acoustic wave) / Layered SAW (Surface acoustic wave) device / MEMS (Micro-electro-mechanical systems)}

\begin{abstract}
In this paper, we investigate the band gaps of micro-machined air/silicon phononic structures both theoretically and experimentally. Based on the plane wave expansion method, dispersion relations of the surface and bulk modes with square lattices in air/silicon two-dimensional phononic structures are calculated and discussed. Band gap widths due to the filling fraction and temperature variation are also analyzed. On the experimental side, generation and reception of high frequency surface acoustic wave in this band structure are realized by a pair of interdigital transducers (IDT) with frequency around $200 \mathrm{MHz}$. Details of the fabricating process of the phononic structure and the high frequency surface wave generating and receiving IDTs are given. The results demonstrate clearly the existence of SAW band gap in the micro-machined phononic structure and this study may serve as a basis for studying the band gap of SAW in micro-machined phononic structure with the dimension in the order of micrometer and find applications in the RF SAW devices.
\end{abstract}

\section{Introduction}

In recent years, application of photonic crystals is an exciting topic for multiple purposes, for example, DWDM (dense wavelength division multiplexing) filters, line defect waveguides [1], sharp bending of light [2], and etc. One-dimensional (comb-like structures), two-dimensional (cylinder arrays in different substrates) and three-dimensional (spherical inclusions in a host matrix) photonic crystal structures have been proposed and demonstrated a variety of fundamental and practical interests [3, 4]. This has led to a rapidly growing interest in the analogous acoustic effects in periodic elastic structures called the phononic crystals. For describing the different kinds of elastic/acoustic waves or the frequency ranges, the phononic crystals can also be called the sonic crystals, ultrasonic crystals or liquid phononic crystals for fluid/fluid band

\footnotetext{
* Correspondence author (e-mail: wutt@ndt.iam.ntu.edu.tw)
}

structure. The repetitive structures made up of different materials can prevent elastic waves from passing by at some specific angles or in certain frequency bands.

For the common knowledge within the physics community, there are bulk and surface acoustic waves (BAW and SAW) in conducting the elastic/acoustic waves in the elastic media. For research in band gaps of BAW modes of phononic structures, the mixed and transverse polarization modes have been carried out using plane wave expansion (PWE) method [5-15]. The effects of the orientation of square rods on the acoustic band gaps in a two-dimensional phononic crystal (solid/air) were discussed [10-13]. In conducting the acoustic waves in the mixed system (fluid and solid), the modified PWE method was used to predict the phenomena between fluid and solid [10, 1215]. Except for the PWE method, the multiple scattering theory was applied to study the band gaps of bulk wave properties in three-dimensional periodic acoustic composites and the band structure of a phononic crystal consisting of complex and frequency dependent Lame' coefficients [16-19]. The finite difference time domain method (FDTD) was applied to interpret the experimental data of two-dimensional systems consisting of cylinders of fluids $(\mathrm{Hg}$, air, and oil) inserted periodically in a finite slab of Al host [20]. The FDTD was also used to calculate the periodic solid-solid, solid-liquid, and solid-vacuum composites [21]. The coupling characteristics of localized phonons in photonic crystal fibers were investigated theoretically using the FDTD method [22]. The experimental evidence for the existence of absolute acoustic band gaps (BAW modes) have also been investigated [20, 23-28]. Vasseur et al. [23, 24] compared the experimental measurement of acoustic transmission through triangular arrays of two-dimensional periodic structures with that of the theoretical calculations. Montero de Espinosa et al. [25] accomplished the experimental demonstration that observing ultrasonic band gap of longitudinal wave in square periodic structures. Torres et al. [26] reported the ultrasonic wedges for elastic wave bending and splitting without requiring a full band gap. Russell et al. [27] detected and controlled the high frequency (about $20 \mathrm{MHz}$ ) sound in a dual-core square-lattice photonic crystal fibre perform using the interferometric set up. Ho et al. [28] demonstrated a class of sonic shield materials based on the locally resonant microstructures and showed that a broad- 
band $(200-500 \mathrm{~Hz})$ sound shield has been achieved experimentally. Recently, the focusing, anomalous, and negative refraction of ultrasound waves in phononic crystals were also reported $[29,30]$.

Investigations into surface wave properties of solids in which the periodic modulation occurs on the traction-free surface did not take place until quite recently [31-37]. The calculations for surface waves on a square and hexagonal superlattice consisting of cubic (AlAs/GaAs) and isotropic ( $\mathrm{Al} /$ polymer) materials were reported [31, 32]. Recently, Wu et al. [33] studied the phononic band gaps of both of the surface and bulk acoustic modes in twodimensional phononic structures consisting of general anisotropic materials. Some literatures aimed at studying the surface acoustic waves in phononic structures experimentally [34-38]. Vinces et al. [34, 35] conducted an experimental study on the surface waves generated by a linefocus acoustic lens at the water-loaded surfaces of a number of two-dimensional superlattices that intersect the surface normally. The propagation of Scholte-like acoustic waves at the liquid-loaded surfaces of periodic structures has also been studied [36]. Rayleigh-wave attenuation in a semi-infinite two-dimensional elastic-band-gap crystal and surface state phenomena in linear and point defects were reported and discussed [37, 38].

Although many theoretical and experimental studies have been done on two-dimensional periodic structures up to now, the experimental studies existed in the literatures are with the dimension in the order of $\mathrm{mm}$ and a few tens of micrometers while the frequency in the order of $\mathrm{kHz}$ and a few tens of $\mathrm{MHz}$ ranges. Toward the application of phononic crystals to the silicon based MEMS components, such as low insertion loss filter, micro acoustic channel and etc., it is necessary to reduce the size to the micrometer (or even further in the nanometer) scale and the frequency in the ranges from couple hundred $\mathrm{MHz}$ to couple GHz. In this paper, we investigate the band gaps of micro-machined air/silicon phononic structures both theoretically and experimentally. Based on the modified PWE method adopted in Ref. [33], dispersion relations of the surface and bulk modes with square and hexagonal lattices in air/silicon two-dimensional phononic structures are calculated and discussed. Band gap widths due to variation of the filling fraction and temperature are also analyzed. The results are then used to design the dimensions and the lattice arrangement of the phononic structures in MEMS fabricating process. To further integrate with the MEMS applications, the CMOS processing techniques are followed to fabricate the two-dimensional phononic structures and the layered SAW devices. A silicon wafer is chosen as the substrate material and combined with an array of cylindrical holes to form a phononic structure. In order to generate surface acoustic waves in the silicon substrate, piezoelectric $\mathrm{ZnO}$ thin film is sputtered on top of the substrate. A pair of interdigital transducers on the $\mathrm{ZnO}$ thin film thus can generate and receive surface acoustic waves. The insertion losses of the $\mathrm{ZnO} / \mathrm{Si}$ layered SAW systems are measured using the network analyzer. Details of the fabricating process of the phononic structure and the high frequency surface wave generating and receiving IDTs are given.

\section{Acoustic waves in two-dimensional phononic band structure}

In the following calculations, the formulation based on the PWE method presented in Ref. [33] was adopted. A brief description of the theory is given in the following. In an inhomogeneous linear elastic medium with no body force, the equation of motion of the displacement vector $\mathbf{u}(\mathbf{r}, t)$ can be written as

$$
\varrho(\mathbf{r}) \ddot{u}_{i}(\mathbf{r}, t)=\partial_{j}\left[C_{i j m n}(\mathbf{r}) \partial_{n} u_{m}(\mathbf{r}, t)\right],
$$

where $\mathbf{r}=(\mathbf{x}, z)=(x, y, z)$ is the position vector; $\varrho(\mathbf{r})$ and $C_{i j m n}(\mathbf{r})$ are the position-dependent mass density and elastic stiffness tensor respectively. We consider a phononic crystal composed of a two-dimensional periodic array ( $x-y$ plane) of material $A$ embedded in a background material $B$. Due to the spatial periodicity, the material constants, $\varrho(\mathbf{x})$ and $C_{i j m n}(\mathbf{x})$ can be expanded in Fourier series with respect to the two-dimensional reciprocal lattice vectors $(\mathrm{RLV}), \mathbf{G}=\left(G_{1}, G_{2}\right)$, as

$$
\begin{aligned}
& \varrho(\mathbf{x})=\sum_{\mathbf{G}} \mathrm{e}^{i \mathbf{G} \cdot \mathbf{x}} \varrho_{\mathbf{G}}, \\
& C_{i j m n}(\mathbf{x})=\sum_{\mathbf{G}} \mathrm{e}^{i \mathbf{G} \cdot \mathbf{x}} C_{\mathbf{G}}^{i j m n},
\end{aligned}
$$

where $\varrho_{\mathbf{G}}$ and $C_{\mathbf{G}}^{i j m n}$ are the corresponding Fourier coefficients.

To utilize the Bloch's theorem and to expand the displacement vector $\mathbf{u}(\mathbf{r}, t)$ in Fourier series for the analyses of the surface and bulk waves, we have

$$
\mathbf{u}(\mathbf{r}, t)=\sum_{\mathbf{G}} \mathrm{e}^{i \mathbf{k} \cdot \mathbf{x}-i \omega t}\left(\mathrm{e}^{i \mathbf{G} \cdot \mathbf{x}} \mathbf{A}_{\mathbf{G}} \mathrm{e}^{i k_{z} z}\right),
$$

where $\mathbf{k}=\left(k_{1}, k_{2}\right)$ is the Bloch wave vector, $\omega$ is the circular frequency, $k_{z}$ is the wave number along the $z$ direction, and $\mathbf{A}_{\mathbf{G}}$ is the amplitude of the displacement vector. We note that as the component of the wave vector $k_{z}$ is equal to zero, Eq. (4) degenerates into the displacement vector of a bulk acoustic wave. On substituting Eqs. (2)-(4) into Eq. (1), and after collecting terms systematically, we obtain the generalized eigenvalue problem as

$$
\left(\mathbf{A} k_{z}^{2}+\mathbf{B} k_{z}+\mathbf{C}\right) \cdot \mathbf{U}=0
$$

where $\mathbf{A}, \mathbf{B}$ and $\mathbf{C}$ are $3 n \times 3 n$ matrices, and are functions of the Bloch wave vector $\mathbf{k}$, components of the two-dimensional RLV, circular frequency $\omega$, the Fourier coefficients of mass density $\varrho_{\mathbf{G}}$ and components of elastic stiffness tensor $C_{\mathbf{G}}^{i j m n} . n$ is the total number of RLV used in the Fourier expansion, and $\mathbf{U}$ is the eigenvector. The expressions of the matrices $\mathbf{A}, \mathbf{B}$ and $\mathbf{C}$ were listed in Ref. [33].

By applying the surface wave conditions and the traction free boundary conditions on the surface, the dispersion relation for the surface waves propagating in the twodimensional phononic crystals, with both of the filling and background materials belonging to the triclinic system, can be obtained [33]. When $k_{z}$ in Eq. (5) is equal to zero, the equation degenerates into the eigenvalue problem of the bulk waves as

$$
\mathbf{C} \cdot \mathbf{U}=0 \text {. }
$$


The dispersion relations of the bulk waves propagating in the two-dimensional phononic crystals can be obtained by setting the determinant of matrix $\mathbf{C}$ equal to zero.

For the analysis of temperature effect on the band gaps of SAW and BAW modes in phononic crystals, the material constants, $\varrho(\mathbf{x})$ and $C_{i j m n}(\mathbf{x})$ in Eqs. (1), (2) and (3) can be expanded at temperature $T$ and in Fourier series. In this paper, air and silicon are utilized as both of the cylinder and the background materials respectively. From the elastic constants at room temperature $\left(25^{\circ} \mathrm{C}\right)$, the temperaturedependent elastic constants of the material, silicon, can be obtained by using [39]

$$
\begin{aligned}
X(T) \approx X\left(T_{0}\right)[ & {\left[1+\frac{1}{X\left(T_{0}\right)} \frac{\partial X}{\partial T}\left(T-T_{0}\right)\right.} \\
& \left.+\frac{1}{2 X\left(T_{0}\right)} \frac{\partial^{2} X}{\partial T^{2}}\left(T-T_{0}\right)^{2}+\ldots\right],
\end{aligned}
$$

where $T_{0}$ is $25^{\circ} \mathrm{C}$, and $X\left(T_{0}\right)$ is the elastic constant evaluated at room temperature. $\frac{1}{X\left(T_{0}\right)} \frac{\partial X}{\partial T}$ and $\frac{1}{2 X\left(T_{0}\right)} \frac{\partial^{2} X}{\partial T^{2}}$ are the first and the second order temperature coefficients respectively. $T$ is increased from $0{ }^{\circ} \mathrm{C}$ to $100{ }^{\circ} \mathrm{C}$. The first order temperature coefficient of the density reads

$$
\frac{1}{\varrho\left(T_{0}\right)} \frac{\partial \varrho}{\partial T}=-\left(\alpha_{11}+\alpha_{22}+\alpha_{33}\right),
$$

where $\alpha_{11}, \alpha_{22}$, and $\alpha_{33}$ are the expansion coefficients along the $x, y$, and $z$ axis respectively.

In this paper, we only consider the first order temperature coefficients and the expansion coefficients of silicon and air. The related constants of the first order temperature coefficients of silicon can be found in Ref. [40] and the thermodynamic properties (density and sound speed) of air can be found in Ref. [41]. It is worth noting that the filling fraction and the effects of the thermal stresses arising from the thermal expansion mismatch between the superlattice components when the temperature is varied are neglected for the case of air/solid band structure.

\section{The band gaps of SAW in the air/silicon band structure}

In this study, we consider a phononic crystal consisting of silicon substrate and square arrays of air cylindrical holes. The modified PWE method can be well approximately used because of the high-density contrast between solid and air [10, 12-15]. It is well-known that the high density contrast between solid and air leads to unexpected flat bands in the band structure. We get round this problem by adopting the technique shown in Ref. [10]. The flat bands can be removed by taking an artificial transverse velocity inside the fluid. Instead of postulating a purely longitudinal behavior of the solid [12-15], we also give an artificial transverse character to the fluid [10]. Figure 1 shows the results of SAW and BAW modes propagating on the surface of a two-dimensional air/silicon phononic structure. The vertical axis is the frequency in units of $\mathrm{MHz}$ and the horizontal axis is the reduced wave vector $\left(k^{*}=k a / \pi\right)$. Where " $a$ " is the distance between two cylinders and $k$ is the wave number. In the calculations, the

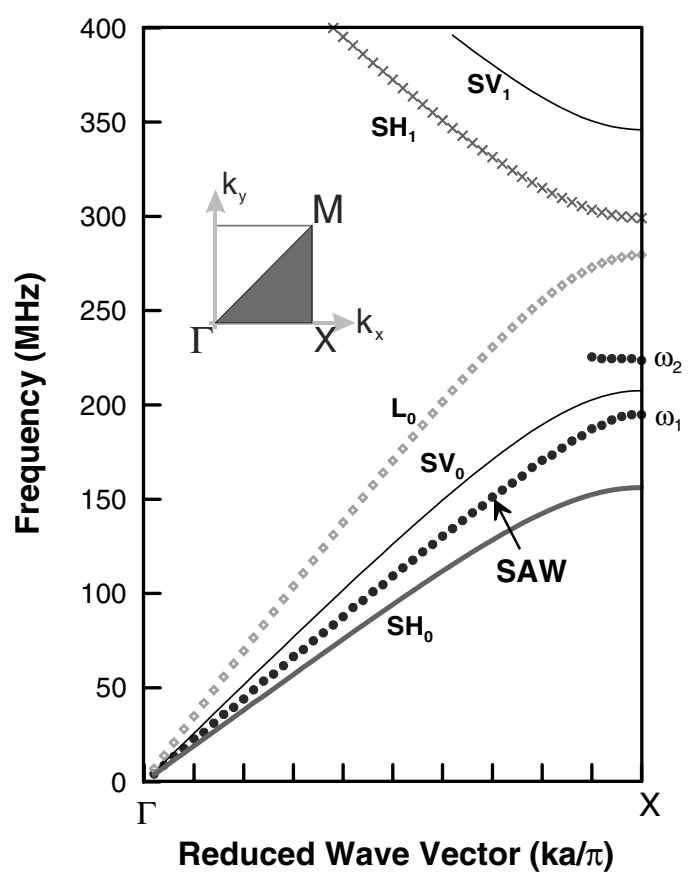

Fig. 1. The dispersion relations of the SAW and BAW modes along the $\Gamma-X$ boundary of the irreducible part of the Brillouin zone with filling ratio $\mathrm{ff}=0.283$. The system of circular rods (air) embedded in a background material (silicon) with square lattice and the lattice constant is $10 \mu \mathrm{m}$.

$x-y$ plane is parallel to the (001) plane and the $x$ axis is parallel to the [100] direction of the silicon. The filling fraction (ff) of the primitive unit cell is $\mathrm{ff}=0.283(\mathrm{r} /$ $a=0.3, a=10 \mu \mathrm{m})$. The irreducible Brillouin zone of the air/silicon phononic structure with square lattice is shown in the inset of Fig. 1. As shown in Fig. 1, along the $\Gamma-X$ boundary of the irreducible Brillouin zone, the fundamental and the higher surface wave modes are belonging to the normal SAW modes (solid circles). At the $X$ point of the irreducible Brillouin zone, the first frequency gap is located between 194.64 MHz and 223.53 MHz. As a preliminary study of SAW band gap in micro-machined phononic structure, we choose the [100] direction as the testing example. For comparison, the dispersion relations of the bulk modes are also shown in Fig. 1. The thin solid lines represent the fundamental $\left(\mathrm{SV}_{0}\right)$ and the higher shear vertical modes $\left(\mathrm{SV}_{1}\right)$. The square symbols are those for the fundamental modes $\left(\mathrm{L}_{0}\right)$. The bold solid lines represent the fundamental shear horizontal modes $\left(\mathrm{SH}_{0}\right)$, while the lines with " $\times$ " symbols represent the higher shear horizontal modes $\left(\mathrm{SH}_{1}\right)$.

The frequencies $\omega_{1}, \omega_{2}$ and the band gap widths $\left(\Delta \omega=\omega_{2}-\omega_{1}\right)$ due to the filling fraction are analyzed in Fig. 2. Shown in Fig. 2, the vertical axis is the frequency in units of $\mathrm{MHz}$ and the horizontal axis is the filling fractions (ff $=\pi r^{2} / a^{2}$ ). The solid circles represent the fundamental and higher SAW modes while the open circles with dash lines represent the band gap widths of SAW modes at the $X$ point. It is worth noting that the frequencies $\omega_{1}$ and $\omega_{2}$ decrease as the filling fraction of the band structure rises. The band gap widths $\Delta \omega$ increase as the filling fraction rises and reach the maximum value at about ff $=0.48$. 


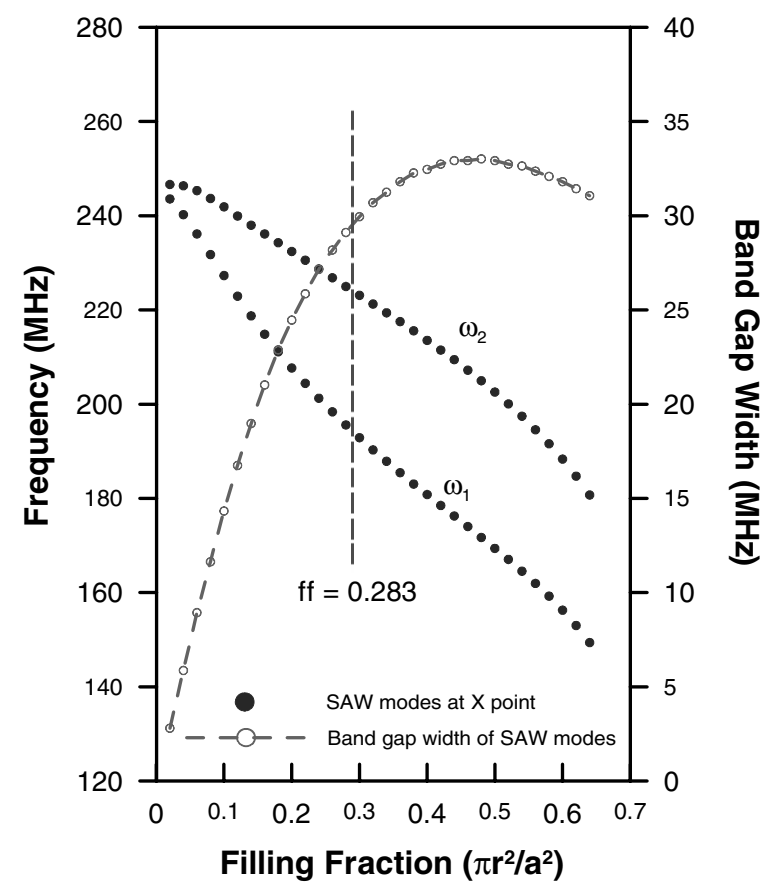

Fig. 2. The variations of the frequencies $\omega_{1}, \omega_{2}$ and the band gap widths due to the filling fraction. The band gap width is defined as $\Delta \omega=\omega_{2}-\omega_{1}$.

On the other hand, the frequencies $\omega_{1}, \omega_{2}$ and the band gap widths of SAW modes at the $\mathrm{X}$ point due to the temperature variation are considered in Fig. 3. In this paper, we increase the temperature of the band structure from 0 to 100 degrees centigrade. The elastic properties and densities of the silicon from $0{ }^{\circ} \mathrm{C}$ to $100{ }^{\circ} \mathrm{C}$ utilized in this example are calculated from Eqs. (7) and (8). The relative elastic properties of air from $0{ }^{\circ} \mathrm{C}$ to $100{ }^{\circ} \mathrm{C}$ are calculated from the data of densities and sound speeds in Ref. [41]. The vertical axis is the frequency in unit of $\mathrm{MHz}$ and the horizontal axis is the temperature of the band structure. In
Fig. 3, the frequencies $\omega_{1}, \omega_{2}$ decrease as the temperature of silicon rises while the band gap widths $\Delta \omega$ also decrease as the temperature of silicon rises. In this study, we found that the band gap widths of the propagating modes can be enlarged or reduced by adjusting not only the filling fraction but also the temperature effect. Furthermore, with the same dimensions of the lattice constant and the radius of cylinders, we also calculated the variations of band gaps of the air/silicon band structure in hexagonal lattice. We found that the band gap width of SAW modes propagating along $x$ axis has the maximum value when the band structure is adjusted in square lattice.

\section{Experimental evidence of SAW band gaps}

To design the dimensions of the phononic structures in MEMS fabricating process, we take the distance between two cylinders " $a$ " equaling to $10 \mu \mathrm{m}$ and the radius of cylinder $r$ equaling to $3 \mu \mathrm{m}$. The band gap along $\Gamma-X$ direction of the two-dimensional air/silicon phononic crystals is located between $194.64 \mathrm{MHz}$ and $223.53 \mathrm{MHz}$. Shown in Fig. 2, the vertical dash line indicates the locations of the frequencies $\omega_{1}, \omega_{2}$ and the band gap widths of SAW modes at $\mathrm{ff}=0.283$. Figure 4 shows the schematic diagram of the layered SAW system. Six rows of cylinders are located between the IDT pair. The layered SAW device fabrication is prior to the drilling of the cylindrical holes in order to avoid deposition of thin film existing on the cylindrical area.

In the following, we describe the procedures for fabricating the two-dimensional phononic crystals and the SAW device. The fabricating process is divided into four major steps. At the outset, the RF magnetron sputtering system with zinc oxide target is used to deposit $\mathrm{ZnO}$ thin film on the silicon substrate. The target is sintered stoichiometric $\mathrm{ZnO}$ with $99.9 \%$ purity, and its dimensions are
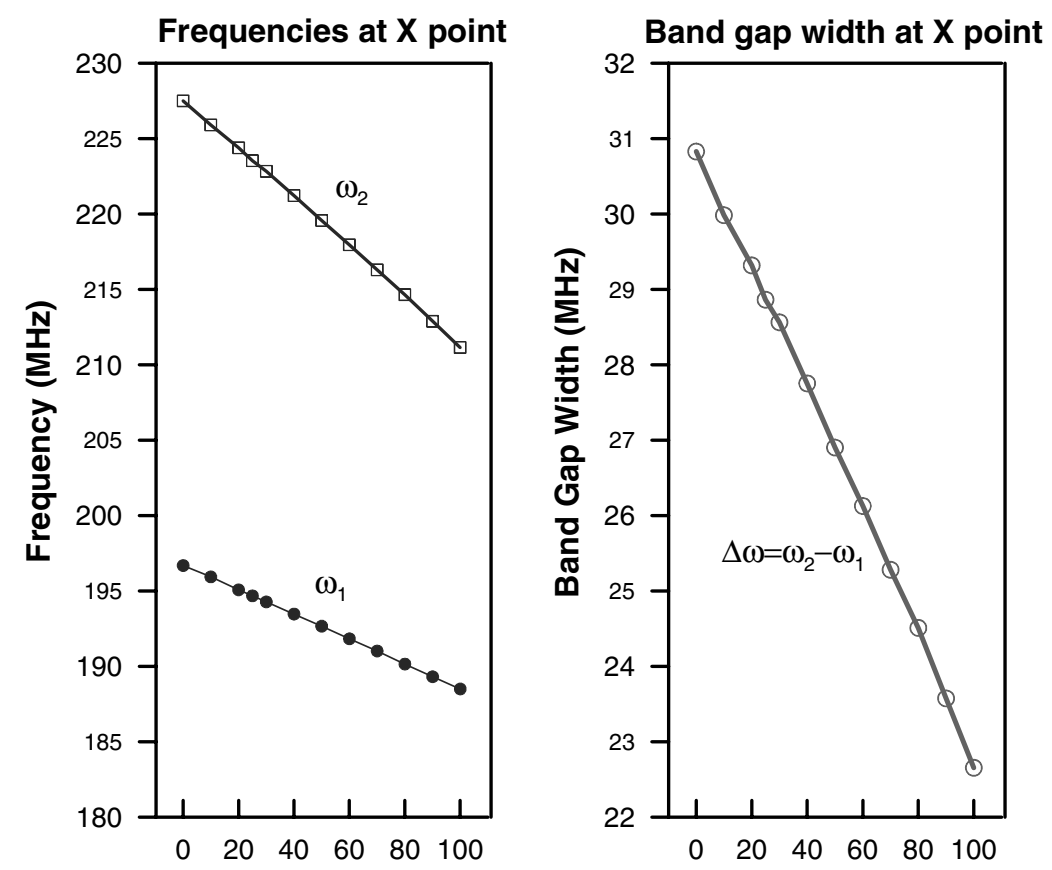

Fig. 3. The frequencies $\omega_{1}, \omega_{2}$ and the band gap widths of SAW modes in the air/silicon phononic structure with cylinder: air $\left(0{ }^{\circ} \mathrm{C}-100{ }^{\circ} \mathrm{C}\right)$ and base: silicon $\left(0^{\circ} \mathrm{C}-\right.$ $100{ }^{\circ} \mathrm{C}$ ).

Temperature of Si and air $\left({ }^{\circ} \mathrm{C}\right)$ 


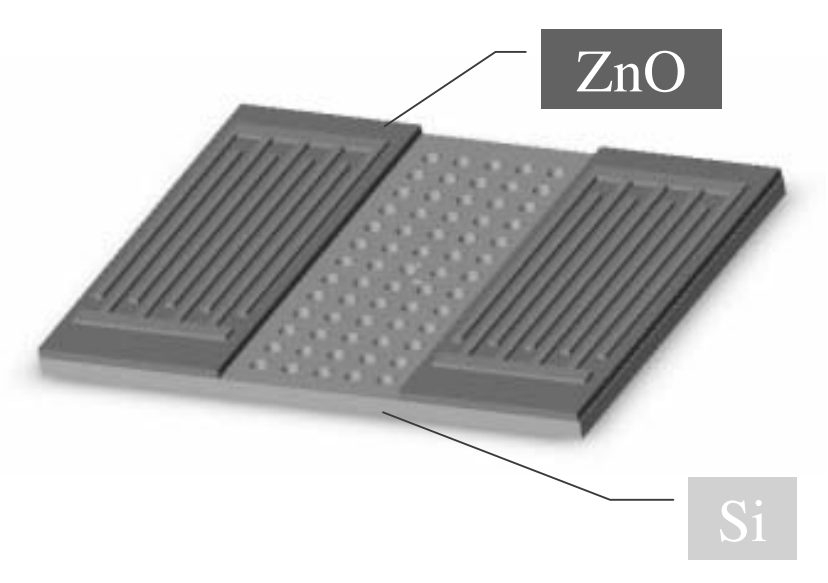

Fig. 4. Schematic diagram of the layered SAW system.

$100 \mathrm{~mm}$ in diameter and $3 \mathrm{~mm}$ in thickness. The distance between the target and the substrate is $13 \mathrm{~cm}$ to make sure of the uniformity of thickness. The thickness of $\mathrm{ZnO}$ thin film is about $1.7 \mu \mathrm{m}$.

Secondly, the layered IDTs are fabricated by using the lift-off process. The advantage of using the lift-off process is that it can prevent patterns from under cutting effect and have better transformation from the photo mask. Both of the transmitting and receiving IDTs consist of 50 pairs of IDT fingers. The thickness of the IDT electrode measured by a surface profiler is about $1950 \AA$. Thirdly, the $\mathrm{ZnO}$ thin film between two IDTs is removed with etching process in order to fabricate the cylindrical holes. The etching time of the $\mathrm{ZnO}$ film by the mixture, $\mathrm{H}_{3} \mathrm{PO}_{4}: \mathrm{HAc}: \mathrm{H}_{2} \mathrm{O}=1: 1: 10$ solution, is about two to four minutes. The final step is to manufacture the two-dimensional periodic arrays of air cylinders. Since the energy of SAW is mostly confined to a depth of one to two wavelengths of the SAW, the depths of the two-dimensional periodic cylinders are fabricated at least $80 \mu \mathrm{m}$. The ICP (Inductively Coupled Plasma-reactive) ion etching is utilized to fabricate the arrays of air cylinders. Different from the traditional RIE etching (Reactive Ion Etching), ICP etching has relatively high etching rate and can reduce lateral etching effectively. Besides, ICP etching can be used to fabricate high aspect ratio structures. In order to protect those finished patterns from the ICP etching, a thicker photo-resist AZP 4620 is used instead of the photo-resist S1813. The depth of the cylindrical holes is around $90 \mu \mathrm{m}$ which is revealed by sectioning the sample and measuring from the SEM image.

Figure 5 shows the SEM image of part of the fabricated air/silicon phononic structure. The image shows that both of the IDTs and the arrays of air cylinders are well fabricated, and the six rows of the air cylinders are located between the two IDTs. The diameter of the cylinder is very close to the designed one, i.e., diameter $=6 \mu \mathrm{m}$.

To demonstrate the band gap of SAW in air/silicon phononic crystal, two layered SAW systems (two IDTs and one delay line) are fabricated. One is located in the band gap and the other one is not. The wavelengths of the two layered SAW systems are $32.8 \mu \mathrm{m}$ and $21.6 \mu \mathrm{m}$ respectively. For comparison, two layered SAW systems without the phononic structure in the silicon delay line are

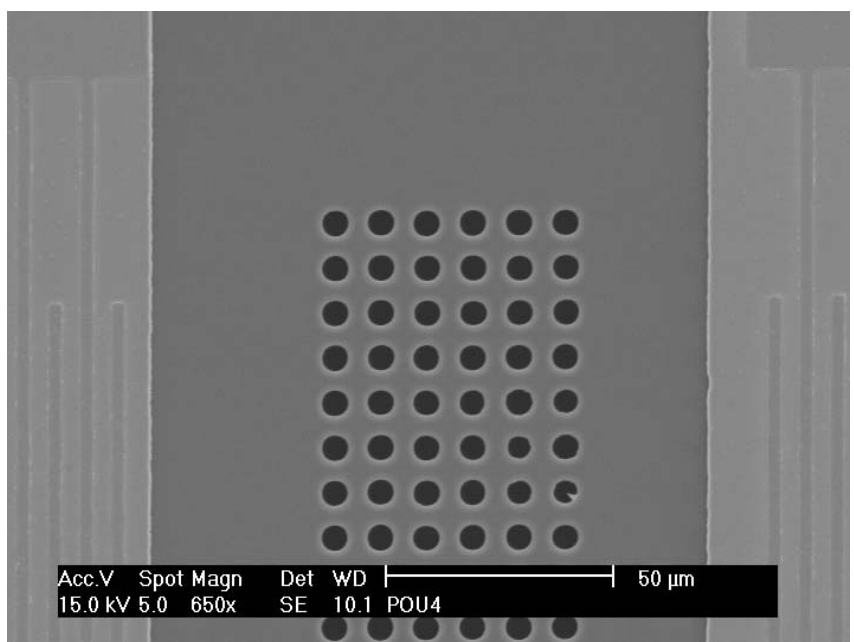

Fig. 5. SEM image displays the layered SAW system.

also fabricated. Except for the phononic structure, all the other parameters of the layered SAW systems are the same as what mentioned above.

Shown in Fig. 6 is the measuring insertion loss of the layered SAW systems with wavelength $32.8 \mu \mathrm{m}$. The vertical axis is the insertion loss in units of $\mathrm{dB}$ and the horizontal axis is the frequency in units of $\mathrm{MHz}$. The $\mathrm{S}_{21}$ parameter is measured from the network analyzer as the insertion loss. The insertion loss is usually expressed in dB. In Fig. 6, the dashed and solid lines are the insertion loss of the SAW systems with and without air/silicon phononic structure respectively. The result shows that the center frequency of the SAW system is around $137 \mathrm{MHz}$, which is located outside the band gap, i.e., $194.64 \mathrm{MHz}$ and $223.53 \mathrm{MHz}$. In addition, we observe that most of the SAW energy transmitted through the phononic structure with very little difference in the insertion loss for the two SAW systems.

On the other hand, the measuring insertion loss of the layered SAW systems with wavelength $21.6 \mu \mathrm{m}$ is shown

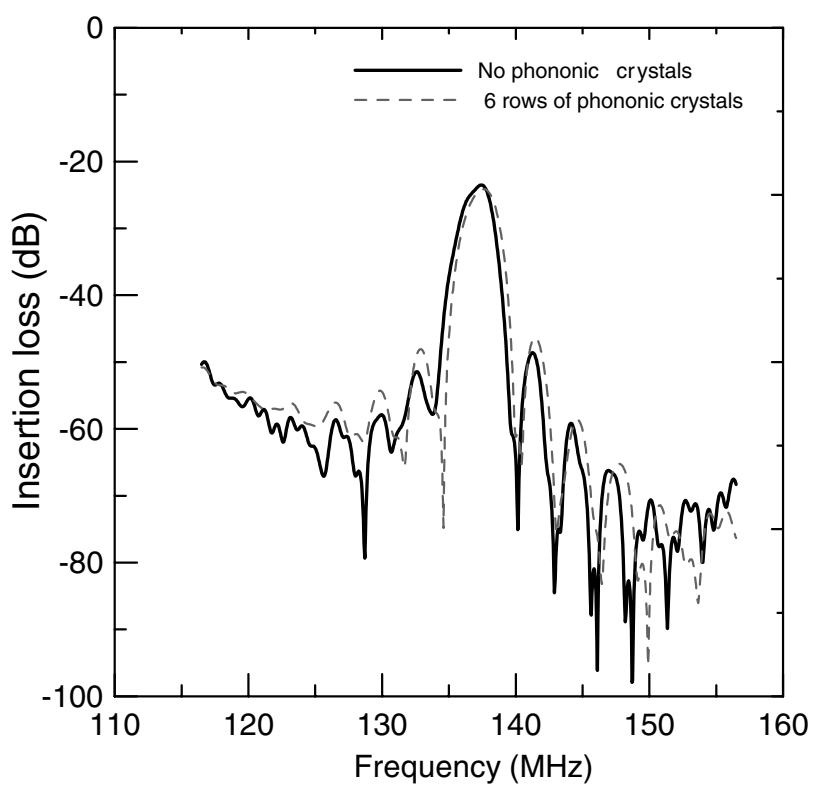

Fig. 6. Frequency responses with and without phononic structure for $\lambda=32.8 \mu \mathrm{m}$. 


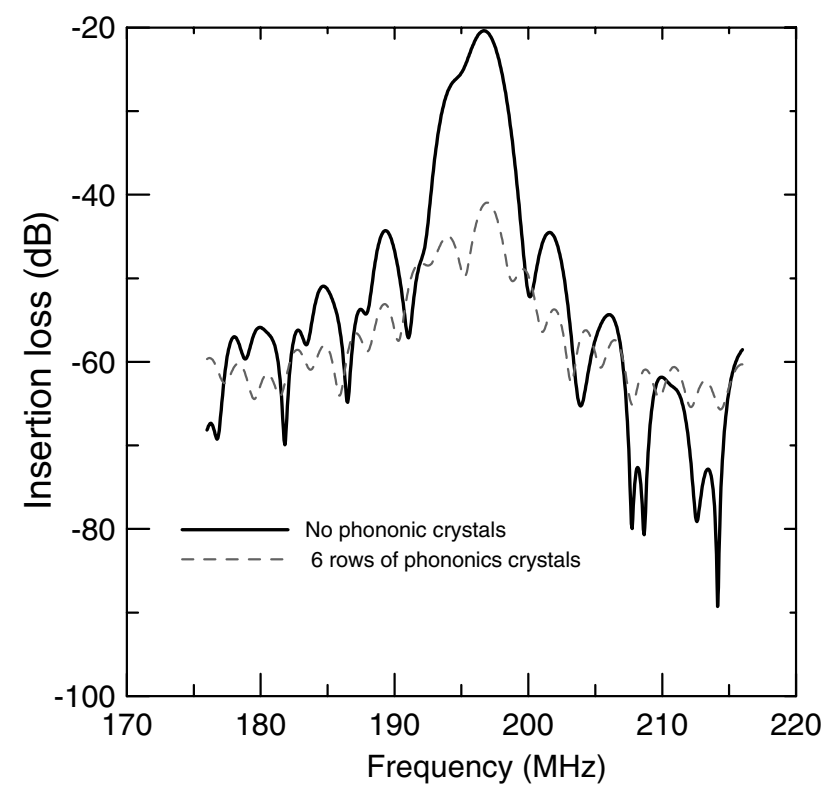

Fig. 7. Frequency responses with and without phononic structure for $\lambda=21.6 \mu \mathrm{m}$.

in Fig. 7. The result shows that the center frequency of the SAW system is around $197 \mathrm{MHz}$, which is located inside the band gap. In this case, we observe that most of the SAW energy is blocked by phononic structure. We also found that the stop band seems to start from $190 \mathrm{MHz}$ instead of the calculated one 194.64 MHz. Nevertheless, the experimental results do demonstrate the existence of a SAW band gap around this frequency range.

\section{Summary and conclusions}

The salient features of the air/silicon phononic band structure with square lattice arrangement have been reported in this paper. In summary, we have presented for the first time an experimental study on the propagation of high frequency SAW in micro-machined two-dimensional phononic structures with the dimensions in the order of micrometer scales. $\mathrm{ZnO} / \mathrm{Si}$ layered SAW systems with and without air/silicon phononic structure are designed and fabricated using the MEMS technique. Details of the fabricating process of the phononic structure and the high frequency surface wave generating and receiving systems are discussed. The insertion losses of the layered SAW systems are measured and the results are demonstrated clearly the existence of SAW band gap in the micro-machined phononic structure. We note that the results of this study may serve as a basis for studying the band gap of SAW in CMOS fabricated phononic structure with the dimension in the order of micrometer and further find applications in the RF SAW devices and MEMS components. The temperature effect on the band gaps of SAW and BAW modes in the phononic structure is quite obvious [42] and the experimental evidence awaits further study.

Acknowledgments. The authors thank the National Science Council (NSC92-2212-E-002-058) and the NTU-ITRI center of Taiwan for financial support.

\section{References}

[1] Lončar, Marko; Doll, Theodor; Vučković, Jelena; Scherer, Axel: Design and fabrication of silicon photonic crystal optical waveguides. J. Lightwave Technology 18 (2000) 1402-1411.

[2] Mekis, A.; Chen, J. C.; Kurland, I.; Fan, S.; Villeneuve, P. R.; Joannopoulos, J. D.: High transmission through sharp bands in photonic crystal wave guides. Phys. Rev. Lett. 77 (1996) 3787.

[3] Johnson, S. G.; Joannopoulos, J. D.: PHOTONIC CRYSTALS: The road from theory to practice. Kluwer academic publishers, Boston 2003.

[4] Joannopoulos, J. D.; Meade, R. D.; Winn, J. N.: Photonic Crystals: Molding the flow of light. Princeton University Press, Princeton, NJ 1995.

[5] Kushwaha, M. S.; Halevi, P.; Dobrzynski, L.; Djafari-Rouhani, B.: Acoustic Band Structure of Periodic Elastic Composites. Phys. Rev. Lett. 71 (1993) 2022-2025.

[6] Kushwaha M. S.; Halevi, P.: Band-gap engineering in periodic elastic composites. Appl. Phys. Lett. 64 (1994) 1085-1087.

[7] Kushwaha, M. S.; Halevi, P.; Martinez, G.; Dobrzynski, L.; Djafari-Rouhani, B.: Theory of acoustic band structure of periodic elastic composites. Phys. Rev. B49 (1994) 2313-2322.

[8] Vasseur, J. O.; Djafari-Rouhani, B.; Dobrzynski, L.; Kushwaha, M. S.; Halevi, P.: Complete acoustic band gaps in periodic fibre reinforced composite materials: the carbon/epoxy composite and some metallic systems. J. Phys.: Condens. Matter 6 (1994) 8759-8770.

[9] Wilm, M.; Khelif, A.; Ballandras, S.; Laude, V.: Out-of-plane propagation of elastic waves in two-dimensional phononic bandgap materials. Phys. Rev. E67 (2003) 065602.

[10] Goffaux, C.; and Vigneron, J. P.: Theoretical study of a tunable phononic band gap system. Phys. Rev. B64 (2001) 075118.

[11] Wu, Fugen; Liu, Zhengyou; Liu, Youyan: Acoustic band gaps in 2D liquid phononic crystals of rectangular structure. J. Phys. D35 (2002) 162-165.

[12] Wu, Fugen; Liu, Zhengyou; Liu, Youyan: Acoustic band gaps created by rotating square rods in a two-dimensional lattice. Phys. Rev. E66 (2002) 046628.

[13] Li, Xiaoling; Wu, Fugen; Hu, Hefei; Zhong, Shao; Liu, Youyan: Large acoustic band gaps created by rotating square rods in twodimensional periodic composites. J. Phys. D: Appl. Phys. 36 (2003) L15-L17.

[14] Sigalas, M. M.; Economou, E. N.: Attenuation of multiple-scattered sound. Europhys. Lett. 36 (1996) 241-246.

[15] Kushwaha M. S.; Halevi, P.: Stop-bands for periodic metallic rods: Sculptures that can filter the noise. Appl. Phys. Lett. 70 (1997) 3218-3220.

[16] Kafesaki, M.; Economou, E. N.: Multiple-scattering theory for three-dimensional periodic acoustic composites. Phys. Rev. B60 (1999) 11993.

[17] Psarobas, I. E.; Stefanou, N.: Scattering of elastic waves by periodic arrays of spherical bodies. Phys. Rev. B62 (2000) 278.

[18] Liu, Zhengyou; Chan, C. T.; Sheng, Ping: Elastic wave scattering by periodic structures of spherical objects: Theory and experiment. Phys. Rev. B62 (2000) 2446.

[19] Mei, Jun; Liu, Zhengyou; Shi, Jing; Tian, Decheng: Theory for elastic wave scattering by a two-dimensional periodical array of cylinders: An ideal approach for band-structure calculations. Phys. Rev. B67 (2003) 245107.

[20] Garica-Pablos, D.; Sigalas, M.; Montero de Espinosa, F. R.; Torres, M.; Kafesaki, M.; Garcia, N.: Theory and Experiments on Elastic Band gaps. Phys. Rev. Lett. 84 (2000) 4349.

[21] Tanaka, Y.; Tomoyasu, Y.; Tamura, S.: Band structure of acoustic waves in phononic lattices: Two-dimensional composites with large acoustic mismatch. Phys. Rev. B62 (2000) $7387-$ 7392.

[22] Khelif, A.; Djafari-Rouhani, B.; Laude, V.; Solal, M.: Coupling characteristics of localized phonons in photonic crystal fibers. J. Appl. Phys. 94 (2003) 7944-7946.

[23] Vasseur, J. O.; Deymier, P. A.; Frantziskonis, G.; Hong, G.; Djafari-Rouhani B.; Dobrzynski, L.: Experimental evidence for the existence of absolute acoustic band gaps in two-dimensional periodic composite media. J. Phys.: Condens. Matter 10 (1998) 6051-6064. 
[24] Vasseur, J. O.; Deymier, P. A.; Chenni, B.; Djafari-Rouhani, B.; Dobrzynski, L.; Prevost, D.: Experimental and theoretical evidence of absolute acoustic band gaps in two-dimensional solid phnonic crystals. Phys. Rev. Lett. 86 (2001) 3012-3015.

[25] Montero de Espinosa, F. R.; Jimenez, E.; Torres, M.: Ultrasonic Band Gap in a Periodic Two-Dimensional Composite. Phys. Rev. Lett. 80 (1998) 1208-1211.

[26] Torres, M.; Montero de Espinosa, F. R.; Aragón, J. L.: Ultrasonic Wedges for Elastic Wave Bending and Splitting without Requiring a Full Band Gap. Phys. Rev. Lett. 86 (2001) 42824285.

[27] Russell, P. St. J.; Marin, E.; Díez, A.: Sonic band gaps in PCF preforms: enhancing the interaction of sound and light. Optics Express 11 (2003) 2555-2560.

[28] Ho, Kin Ming; Cheng, Chun Kwong; Yang, Z.; Zhang, X. X.; Sheng, Ping: Broadband locally resonant sonic shields. Appl. Phys. Lett. 83 (2003) 5566-5569.

[29] Torres, M.; Montero de Espinosa, F. R.: Ultrasonic band gaps and negative refraction. Ultrasonics 42 (2004) 787-790.

[30] Yang, S.; Page, J. H.; Liu, Z.; Cowan, M. L.; Chan, C. T.; Sheng, Ping: Focusing of sound in a 3D phononic crystal. Phys. Rev. Lett. 93 (2004) 024301.

[31] Tanaka, Y.; Tamura, S.: Surface acoustic waves in two-dimensional periodic elastic structures. Phys. Rev. B58 (1998) 7958.

[32] Tanaka, Y.; Tamura, S.: Acoustic stop bands of surface and bulk modes in two-dimensional phononic lattices consisting of aluminum and a polymer. Phys. Rev. B60 (1999) 13294.

[33] Wu, Tsung-Tsong; Huang, Zi-Gui; Lin, S.: Surface and bulk acoustic waves in two-dimensional phononic crystals consisting of materials with general anisotropy. Phys. Rev. B69 (2004) 094301.

[34] Vines, R. E.; Wolfe, J. P.; Every, A. G.: Scanning phononic lattices with ultrasound. Phys. Rev. B60 (1999) 11871.

[35] Vines R. E.; Wolfe, J. P.: Scanning phononic lattices with surface acoustic waves. Physica B263-264 (1999) 567.

[36] Every, A. G.; Vines R. E.; Wolfe, J. P.: Line-focus probe excitation of Scholte acoustic waves at the liquid-loaded surfaces of periodic structures. Phys. Rev. B60 (1999) 11755.

[37] Meseguer, F.; Holgado, M.; Caballero, D.; Benaches, N. ; Sa'nchez-Dehesa, J.; López, C.; Llinares, J.: Rayleigh-wave attenuation by a semi-infinite two-dimensional elastic-band-gap crystal. Phys. Rev. B59 (1999) 12169-12172.

[38] Torres, M.; Montero de Espinosa, F. R.; Garica-Pablos, D.; Garcia, N.: Sonic Band Gaps in Finite Elastic Media Surface States and Localization Phenomena in Linear and Point Defects. Phys. Rev. Lett. 82 (1999) 4282-4285.

[39] Oliner, A. A.: Acoustic Surface Waves-topics in Applied Physics 24. Springer-Verlag Berlin Heidelberg, New York, 1978.

[40] McSkimin, H. J.: Measurement of Elastic Constants at Low Temperatures by Means of Ultrasonic Waves-Data for Silicon and Germanium Single Crystals, and for Fused Silica. J. Appl. Phys. 24 (1953) 988-997.

[41] David R. Lide: CRC handbook of chemistry and physics. CRC press 83rd ed. Boca Raton London, NY, (2002-2003).

[42] Huang, Zi-Gui; Wu, Tsung-Tsong: Temperature effects on bandgaps of surface and bulk acoustic waves in two-dimensional phononic crystals. IEEE Trans. Ultrason. Ferroelectr. Freq. Control 52 (2005) 365-370. 